\title{
L'ethnopoétique et l'anthropologie structurale à partir d'un récit de Victoria Howard, Chinook Clakamas
}

\section{Catharine Mason}

\section{OpenEdition}

\section{Journals}

Édition électronique

URL : https://journals.openedition.org/jsa/308

DOI : $10.4000 /$ jsa. 308

ISSN : 1957-7842

Éditeur

Société des américanistes

Édition imprimée

Date de publication : 5 janvier 2004

Pagination : 25-55

ISSN : 0037-9174

Référence électronique

Catharine Mason, «L'ethnopoétique et l'anthropologie structurale à partir d'un récit de Victoria Howard, Chinook Clakamas », Journal de la Société des américanistes [En ligne], 90-1 | 2004, mis en ligne le 05 janvier 2009, consulté le 03 septembre 2022. URL : http://journals.openedition.org/jsa/308 ; DOI : https://doi.org/10.4000/jsa.308 


\title{
L'ETHNOPOÉTIQUE ET L'ANTHROPOLOGIE STRUCTURALE À PARTIR D'UN RÉCIT DE VICTORIA HOWARD, CHINOOK CLACKAMAS
}

\author{
Catharine MASON *
}

L'étude d'un récit de mythe chinook clackamas, énoncé par Victoria Howard au linguiste Melville Jacobs en 1930, fournit la base d'une comparaison entre deux méthodologies d'analyse mythologique. L'approche de l'anthropologie structurale innovée par Claude Lévi-Strauss et appliquée dans son interprétation du texte de Howard par rapport aux autres narrations du même mythe est juxtaposée à une analyse ethnopoétique qui s'appuie sur la logique interne d'un seul récit. Cet article cherche à démontrer la compatibilité de ces deux approches scientifiques à travers des éléments concrets du texte et s'attache, aussi, à souligner leurs objectifs communs, tant pratiques que théoriques, dans l'étude des traditions orales. [MOTS CLÉS : mythe, chinook clackamas, Victoria Howard, ethnopoétique, anthropologie structurale, Claude Lévi-Strauss, Dell Hymes.]

Ethmopoetics and structural anthropology as seen through a narrative by Victoria Howard, Clackamas Chinook. The study of a Clackamas Chinookan myth told by Victoria Howard to the linguist, Melville Jacobs, in 1930 provides the basis for a comparison of two methodologies of myth analysis. The approach of structural anthropology, innovated by Claude Lévi-Strauss and applied in his interpretation of Howard's text with relation to other narrations of this same myth, is placed side by side with an ethnopoetic analysis which is based on the internal logic of an individual narrative. This article seeks to demonstrate the compatibility of these two scientific approaches using concrete elements of the text. It also points to common objectives of these two approaches, both practical and theoretical, in the study of oral tradition. [KEY worDs : myth, Clackamas Chinook, Victoria Howard, ethnopoetics, structural anthropology, Claude LéviStrauss, Dell Hymes.]

Etnopoética y antropología estructural aplicadas a um relato de Victoria Howard, chinook clackaman. El estudio de un relato, de mito chinook clackaman, expuesto por Victoria Howard al linguista Melville Jacobs en 1930, aporta la base de una comparación entre dos metodologías de análisis mitológico. El enfoque de la antropología estructural instaurada por Claude Lévi-Strauss y aplicada a la interpretación del texto de Howard,

* Maison de la recherche en sciences humaines, Université de Caen [cmason@mrsh.unicaen.fr]. Journal de la Société des Américanistes, 2004, 90-1, pp. 25-55. OSociété des Américanistes. 
con relación a las otras narraciones del mismo mito, se yuxtapone con un análisis etnopoético basado en la lógica interna del único relato. Este artículo intenta demostrar la compatibilidad de los dos enfoques científicos, a través de elementos concretos del texto y también, subraya sus objetivos comunes, tanto prácticos, como teóricos, en el estudio de las tradiciones orales. [PALABRAS ClAVES : mito, chinook clackaman, Victoria Howard, etnopoética, antropología estructural, Claude Lévi-Strauss, Dell Hymes.]

Deux travaux ont marqué des étapes importantes dans l'approche stylistique des récits oraux développée par Dell Hymes : In Vain I tried to tell you (1981) et "Language, memory, and selective performance : Cultee's "Salmon's Myth" as twice told to Boas » (1985). Le premier réunit des articles fondateurs de l'ethnopoétique hymesienne à travers l'étude des dispositifs, formules et stratégies poétiques et rhétoriques qui constituent dans leur ensemble la forme versifiée et la compétence performative d'un récit oral ; il établit les postulats qui détermineront par la suite une orientation majeure des écoles ethnopoétiques. Le second affirme non seulement la pertinence de l'analyse de la versification démontrée dans In vain I tried to tell you, mais aussi la différenciation de significations potentielles entre les narrations d'un même mythe. À première vue, la narration de Salmon's Myth, en 1894, par Charles Cultee semble plus élaborée que celle qu'il a faite en 1891 et, d'après Hymes, suggère une amélioration consécutive à trois ans d'exercice de mémoire et de pratique narrative dans son travail avec Franz Boas. Cependant, l'analyse de cette «élaboration », fournie par Hymes, consiste à dégager les choix délibérés du conteur qui configure ses récits selon des stratégies de significations volontaires.

Alors qu'il était encore doctorant à l'université d'Indiana, Hymes contribua largement à la rédaction des « Results of the conference of anthropologists and linguists ». Les résultats de cette conférence décisive dans le développement de l'anthropologie linguistique américaine sont publiés dans International Journal of American Linguistics (1953). La publication inclut les communications finales de Claude Lévi-Strauss et de Roman Jakobson, l'une résumant le point de vue des anthropologues, l'autre celui des linguistes. Originaire du nord-ouest des ÉtatsUnis et spécialiste des langues chinook, Hymes ne pouvait qu'être sensible aux analyses structurales des Mythologiques. Il écrit « je ne cède à personne mon admiration pour Lévi-Strauss au sujet de sa découverte des relations de transformation » et ajoute cependant une critique : « [...] mais c'est un étrange tribut à la linguistique que d'ignorer le langage » (Hymes 1985, p. 395). Lévi-Strauss répond sur ce point et sur d'autres dans un article, intitulé « De la fidélité au texte » (1987), dans lequel il offre une discussion sur les différences entre sa méthode et celle de Hymes, suivie d'une démonstration de l'analyse mythographique prenant comme objet plusieurs versions du Salmon's Myth. Lévi-Strauss (1987, pp. 131-132) conclut ainsi : 
[...] L'analyse structurale que Hymes appellerait probablement théorique puisqu'il lui oppose son "structuralisme pratique ", et que je qualifierais plutôt de comparative, peut seule dégager ces éléments communs - "commonalities » de Dell Hymes - qui constituent la matière première livrée par la tradition à l'artiste créateur. À défaut de connaître la nature de ce matériau et ses contraintes spécifiques, on ne pourrait évaluer justement la façon dont l'artiste l'élabore, le degré de liberté dont il jouit, les limites qu'il ne peut franchir. Mais il est vrai aussi que des transformations indépendantes des goûts et de la volonté du conteur, induites par les caractères du milieu dans lequel a pris forme et s'est développée une culture particulière, risquent d'échapper en partie au mythologue s'il ne reçoit pas l'aide du linguiste pour élucider le sens précis de certains termes et savoir, avant de commencer l'analyse, ce que dit exactement le mythe. On doit donc exclure toute idée de conflit ou de rivalité entre eux. Une approche ne vaut pas mieux que l'autre. Même parallèles, elles ne doivent ni ne peuvent s'ignorer ; et le plus souvent, elles se croisent ou elles convergent.

Nous sommes d'accord avec Lévi-Strauss sur le fait que les deux méthodes ne sont nullement en contradiction. Cependant l'objet de l'ethnopoétique ne se limite pas à définir « le sens précis de certains termes ». Dans l'étude compréhensive du mythe, il est nécessaire, d'un point de vue sociolinguistique, de savoir non seulement ce que dit un mythe, mais aussi ce qu'il fait dans un contexte social réel.

$\mathrm{Si}$, parfois, le linguiste peut fournir à l'anthropologue des précisions lexicales, sa plus grande contribution à une étude de l'homme et de ses sociétés a néanmoins été d'approfondir la connaissance du fonctionnement du récit dans sa représentation et sa négociation continues des valeurs, des attitudes, des rôles sociaux et des règles et codes sociaux. Comme dirait Ernst Cassirer (1973), l'objectif est de comprendre en quoi la pensée mythique établit l'orientation spatiale et spirituelle du monde de l'homme. L'ethnopoétique, tout comme la mythographie comparative, recherche les valeurs exprimées à travers la conceptualisation mythique du monde vécu et le rôle de cette conceptualisation dans l'organisation même du vécu (spatial et spirituel) de l'homme. Que ce soit à partir d'une expression personnelle ou culturelle, immédiate ou lointaine, ce qui importe pour les deux approches, c'est que les valeurs soient extraites d'une orientation concrète de la société étudiée et fournissent ainsi des données empiriques. Lévi-Strauss (ibid., p. 121) constate l'importance de la méthode inductive pour ses analyses :

[...] quant à moi, je n'ai jamais emprunté mes modèles ailleurs : ils me furent imposés par les mythes mêmes. La meilleure preuve en est qu'ils s'éloignent de tous ceux de mes devanciers et qu'avant qu'ils ne ressortent des Mythologiques, ils demeurent insoupçonnés. Ils ont même souvent rencontré l'incrédulité.

C'est aussi le cas pour Hymes. C'est sur ce point que nos deux auteurs confirment leur fidélité au texte, pour reprendre le titre de Lévi-Strauss. L'obscurité de l'ethnopoétique hymesienne est souvent issue de cette fidélité qui amène le linguiste à inventer des termes « non-spécialistes » pour décrire des phénomènes 
stylistiques, une pratique qui peut inciter les chercheurs non anglophones à se plaindre des idiotismes américains et qui, dans le pire des cas, prête à confusion en raison de l'enchevêtrement des trois disciplines (linguistique, anthropologie, critique littéraire) qui induit l'approche ethnopoétique.

Le grand écart entre les approches lévi-straussienne et hymsienne réside précisément dans l'optique empruntée par chacun et dans les questions posées. L'anthropologie structurale nous ouvre les yeux sur les relations structurantes et opérationnelles, voire institutionnelles, entre les grandes pratiques de la vie sociale. Elle étudie les macrostructures de la société qui fournissent les contours et les grilles mêmes de cette vie, alors que celles-ci déterminent les bornes sociales et les valeurs référentielles auxquelles les membres d'une société adhèrent. Le mythe est, pour l'anthropologue, une opération schématique du discours narratif qui interprète le vécu historique et matériel de l'homme et qui permet au scientifique un aperçu de l'intelligibilité entre les instances de narration différentes et espacées aussi bien qu'entre le corpus mythologique et les autres pratiques sociales (organisation sociale, économie, rite).

L'ethnopoéticien emprunte un chemin bien plus microscopique dans son étude du rôle du mythe et de son rapport à la vie sociale. Se positionnant à l'intérieur du texte, cette approche étudie les pratiques formelles du discours narratif, s'intéressant plus particulièrement aux règles, valeurs, opérations et codes communicatifs qui gouvernent la construction du sens et déterminent la logique interne du texte. L'ethnopoéticien s'interroge sur l'acte de la narration en tant que négociation de valeurs traditionnelles par rapport aux besoins, mobiles, exigences, désirs contemporains. De ce point de vue, la fonction poétique, telle qu'elle a été décrite par Roman Jakobson (1973), fait partie intégrante de l'analyse. Tout comme la langue, la poétique est un acte culturellement spécifique. Les structures formelles d'un récit sont des marqueurs essentiels dans l'interprétation du sens. En bref, pour l'anthropologue (structural), le texte représente un ensemble d'éléments engendrés par des codes, des signes et des valeurs antérieurs. Pour l'ethnopoéticien, le texte est un discours formellement construit, spécifique à son contexte et agissant sur celui-ci.

Nous insistons sur le fait que les deux approches étudient le sens que l'homme donne à l'organisation et l'orientation de sa vie. Le projet mené par Lévi-Strauss et poursuivi par Emmanuel Désveaux $(1988 ; 2001)$ consiste à calculer la sommation de toutes les équations extraites des données précisément définies par l'ethnographie américaine. De son côté, Hymes dévoile les processus eux-mêmes de signification en cherchant la logique qui gouverne les dires transcrits par les ethnolinguistes. L'un cherche les liaisons entre les dires et les pratiques mythiques, rituels, sociaux, économiques afin d'identifier les relations et leurs transformations qui forment le sens sous-jacent de ces activités. L'autre met l'emphase sur l'émergence des dires et de l'échange verbal en tant que pratiques dans la création du sens. Ainsi, l'anthropologue éclaire le sens, le linguiste éclaire l'acte de signifier. 
Il est vrai qu'à un certain stade de développement de ces deux approches sur un même mythe, chacune d'elles semble étrangère l'une à l'autre. Cependant, à aucun moment, les analyses de Salmon's Myth fournies par Hymes et par Lévi-Strauss ne se contredisent. Où se trouvent alors les croisements et les convergences dont Lévi-Strauss constate l'existence entre la mythographie comparative et l'ethnopoétique? Dans une étude ethnopoétique de Waskintwa kwaliwi Wakitsimáni išdáquq, nous verrons que ce récit, donné par Victoria Howard en chinook clackamas, présente une sémantique narrative très différente du sens dévoilé par Lévi-Strauss pour l'ensemble des mythes dont il fait partie. Cependant, une comparaison des deux interprétations nous aidera non seulement à constater la compatibilité de leurs méthodes, mais aussi à voir comment elles peuvent se combiner afin d'éclairer le même objet d'étude. Pour ce faire, nous aurons également recours à d'autres méthodes. La narratologie structurale, en particulier, servira d'intermédiaire permettant, sinon de jeter un pont entre les deux approches, tout au moins d'ouvrir une perspective qui englobe un certain nombre de phénomènes mis en évidence par chacune ${ }^{1}$. Les distinctions, par exemple, entre l'événement narratif et celui narré, entre le temps narratif et celui narré facilitent la transition entre le texte comme bloc sémantique et le texte comme performance.

Deux concepts s'imposent avant d'entamer notre analyse. Il s'agit du « mythe » dans l'anthropologie structurale et de la « performance » dans l'ethnopoétique. Sans entrer dans les théories des mythologies, c'est précisément parce que l'histoire de la fin de la cohabitation de deux espèces, histoire à laquelle nous allons prêter attention, possède le statut du « mythe » qu'on peut aller chercher son sens à travers le temps et l'espace. Or; à travers la démonstration d'une armature structurante et de relations transformationnelles, ce statut s'éclaire et se confirme dans sa portée historique, sociale, économique, psychologique, géographique, astronomique, botanique, métaphysique, et ontologique. Mais, sans la performance, c'est-à-dire sans l'interprétation d'un conteur conscient de son héritage culturel en présence d'un ou de plusieurs narrataire(s), ce sens lointain et répandu, et donc mythique, se dissipe. Notre hypothèse est simple : la voix personnelle, constituée dans l'« arène de la performance » ${ }^{2}$ et nourrie d'un symbolisme ancien, véhicule nécessairement un sens culturel transmis à travers les générations; elle s'adapte au contexte immédiat selon le savoir-faire du conteur et les attentes des auditeurs/lecteurs. C'est de ce savoir-faire et de ces attentes culturelles que se préoccupe l'ethnopoétique.

\section{Victoria Howard, La CONTEUSE ET SA NARRATION}

Victoria Howard naquit aux alentours de 1870, à Grand Ronde Reservation en Oregon, où sa grand-mère, chinook clackamas, et ses contemporains furent 
déplacés avec d'autres peuples amérindiens (Molales, Rogue Rivers, Tillamooks) par le gouvernement américain. Le peuple chinook avait occupé les terres près de la rivière Columbia, de la côte ou de l'embouchure jusqu'aux Dalles des Cascade Mountains, depuis environ 2000 ans. La langue clackamas, intelligible aux locuteurs du wishram-wasco, est une langue du Haut Chinook. Victoria Howard a dicté des mythes, des contes, des récits historiques, des autobiographies et des descriptions culturelles principalement en clackamas, mais aussi en chinook jargon. Suite à ses dictées, l'informatrice a aidé son enquêteur pour la traduction de ses paroles en langue anglaise qu'elle parlait (et peut-être lisait) couramment. Ces entretiens avec le linguiste Melville Jacobs en 1929-1930 furent réalisés sur une période de quatre mois à West Linn où Howard habitait avec son époux. Ces transcriptions furent ensuite éditées par Jacobs en 1958 et 1959. Elles constituent le corpus le plus volumineux issu d'une personne de cette aire géographique et contiennent les seules informations disponibles sur la vie de la locutrice.

Elevée par sa grand-mère maternelle qui ne parlait ni anglais ni chinook jargon et qui lui raconta des mythes et contes de sa tradition, Howard fut éduquée à la manière traditionnelle de ses ancêtres. D'autres récits qu'elle a dictés à Jacobs lui avaient été également racontés par sa belle-mère, d'origine clackamas et klikitat sahaptin. Ayant été imprégnée par la tradition orale de la langue et la littérature clackamas, notre conteuse relate ces histoires avec une maitrise stylistique constatée par l'enquêteur (Jacobs 1959b ; 1960) et par des analyses ethnopoétiques (Hymes 1981 ; Mason 1999).

Victoria Howard narra Waskíntwa kwalíwi Wakitsimáni išdáquq (Ourse noire, femme Grizzly' et leurs fils) à Melville Jacobs vers la fin de leur période de travail à West Linn. C'était sa belle-mère qui lui avait appris ce mythe, probablement à Grand Ronde. Les narrations de cette conteuse sont également le fruit d'un travail de collaboration avec le linguiste, enrichissant la documentation sur la culture et la littérature chinook. De toute évidence, l'informatrice fut consciente de cet objectif scientifique et y adhéra pleinement. Il est également évident que Victoria Howard se souciait de la forme de ses narrations, qu'elle ne se satisfaisait pas de fournir des unités référentielles d'informations, des simples séquences d'événements, mais qu'elle configurait ses dires sans doute pendant un instant pour que " chaque détail désire le tout, et comble et réveille ce désir » (Tadié 1994, p. 117).

Encouragée certainement par l'enthousiasme et la compétence du linguiste, Victoria Howard fit d'un grand nombre de ses récits de véritables performances et Waskintwa kwaliwi Wakitsimáni išdáquq figure parmi ses meilleures. L'émergence d'une performance se constate dans l'analyse stylistique, telle que la définit Jean-Pierre Richard (1955, p. 10): "Ce qui signale toute grande œuvre d'art, c'est assurément sa cohérence interne. Entre les divers plans de l'expérience on y voit s'établir des échos, des convergences. Lire, c'est sans doute provoquer ces échos, saisir ces rapports nouveaux, lier des gerbes de convergence». Nous célébrons alors l'achèvement poétique de notre conteuse et cherchons à faire 
connaître la grande qualité artistique de cette poétesse ainsi que de sa culture malheureusement dissipée.

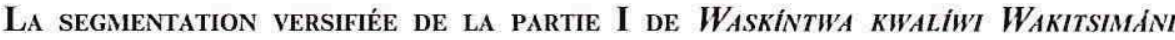 IŠDAQUQ}

Ce texte se divise en trois parties liées par des parallèles rhétoriques, thématiques et symboliques. La première est composé de six actes que nous désignerons par les premières lettres de l'alphabet. L'acte A sert de prologue. Nous admirons, dès le début du récit, la relation entre forme et contenu que notre poétesse a su confectionner. Les cinq premiers vers sont construits sur un équilibre remarquable qui met, subtilement, l'accent sur le déséquilibre déclenchant l'histoire.

GałXílayt díxtka idə'kaqw1.

K'ánidítul,

Askíntwa ičáquq.

K’ánidítuł,

Wakitsimáni ičáquq, lg̀wa"nmikš.

K’wáżí wít’aX Askíntwa lg̀wa'nmikš ičáquq

Wígwa ałXmútXma iłqádutinkš. ${ }^{3}$

Ils habitèrent dans leur seule et unique maison.

De ce côté du feu :

Askíntwa et ses fils.

De ce côté du feu :

Wakitsimáni et ses fils, cinq entre eux.

De même Askíntwa avait cinq fils.

Toute la journée, les dix enfants jouaient ensemble.
A/1

A/1

Nous verrons plus loin en quoi la structure de ce prologue induit le sens narratif.

Les cinq autres actes se réalisent suivant le développement classique de deux modèles événementiels < départ-déroulement-résultat > ; le troisième acte, servant de charnière entre les deux regroupements, correspond au résultat du premier et au départ du deuxième. Dans l'acte B (départ de la séquence), les deux mères, Skíntwa (Ourse Noire) et Kitsimani (Ourse Grizzly), sortent deux fois cueillir des mûres, la première sortie étant décrite en détail par la conteuse tandis que la seconde est racontée en deux lignes, suggérant une simple répétition. L'acte C relate une troisième sortie qui est accompagnée de deux avertissements faits par Skíntwa à son fils : son non-retour signifierait qu'elle a été assassinée par Kitsimani et qu'il lui faut donc se sauver avec ses frères. L'assassinat de Skíntwa fait 
objet de l'acte $\mathrm{D}$ (résultat de la première séquence et départ de la deuxième). L'acte E fait revenir Kitsimani seule de la cueillette, ce qui est observé par Wasguk'mayli, fils aîné de Skíntwa ; l'ogresse cuisine les seins de sa victime, provoquant les pleurs du bébé orphelin. Alors que Kitsimani cache ses actes de meurtre et de mutilation, Wasguk'mayli feint d'ignorer la vérité et dissimule ses intentions.

Gayúčukwdix aġa nákim,

gakłúlxam,

« Ag̉a anugálmama wámšaq.

Wápul yúqəlqt k'áskaš. »

$\mathrm{Ne}$ :' :šqi qa gačúlxam.

Galúya.

Le lendemain matin alors elle lui dit,

elle l'informa,

"Alors je m'en vais chercher votre mère.

Le bébé a pleuré toute la nuit. »

Rien du tout il ne lui dit.

Elle s'en alla.

Le héros tue les enfants de Kitsimani dans l'acte $\mathrm{F}$ (résultat de cette deuxième séquence) et s'enfuit avec ses frères, laissant quatre pièges à destination de l'ogresse afin de détourner son attention et de gagner ainsi du temps.

La deuxième partie du récit, composée de sept actes, comporte trois sousparties qui suivent les deux séquences du modèle événementiel décrit ci-dessus et un septième acte, indépendant. Ces sous-parties, nous le verrons, opèrent également selon le modèle < départ - développement - résultat >. L'acte $\mathrm{G}$ commence par le retour de Kitsimani à son foyer où elle découvre des enfants bouillant dans une soupe. Imaginant que son propre fils aîné a mis les enfants de Skíntwa dans le bouillon - idée prévue par le héros -, elle se met à chanter sa victoire contre la famille ourse. L'acte se termine sur la scène de l'ogresse comprenant qu'il s'agit de ses propres enfants dans le chaudron. La scène est suivie par celle, émouvante, d'une mère en deuil.

Nákim,

« Ádí wígwa ontXdínaya ičxə'mal!

"əčə'xan ičłúdina iłskintwámaX.

Ičla'nalčXm. »

Núlayt,

naXáq’oltgiba, naXłXə'ləmčk.

Sa :' :q'w gagyałq’əlládaba : ičáxan íyaXu iXə'n $\star k$ 'ikWt. « Ú : : : : ičá'xan ! » nákim.

Naga'mquq sa :' : :q'w. 
Elle dit,

«Ah! ah ! toute la journée le bouillon et moi, nous serons en train de nous disputer!

Mon fils a tué les Ours ;

Il les a bouillis pour moi. "

Elle s'assit,

elle sortit la viande (du chaudron),

elle mangea, mangea.

Toute, elle la sortit :

Le bras plié de son fils.

"Oh mon fils! " elle dit.

Elle vomit, tout.

NaXlúxwayt,

«Anyúkšdama íxt ičə'xan. »

Nákta

wáwa gagíyux.

Yutxwi :' :la,

iXk'aya :' : :wola.

Naygə'ltačk,

gagígəlga :

yúmqt.

GagiXíma

Elle pensa,

" J'irai voir celui-là : mon fils. »

Elle y alla,

elle lui parla.

Il était planté là,

Il riait.

Elle l'approcha ;

elle le saisit :

Il est mort.

Elle le coucha.

La chasse contre Wasguk'mayli et ses frères par une mère grizzly furieuse débute dans l'acte $\mathrm{H}$, chasse à nouveau ralentie par le deuxième appât : le chien domestique, instruit par Wasguk'mayli (acte F), cache le trou par lequel les enfants se sont évadés et fait courir l'ogresse dans tous les sens. L'acte I raconte la troisième prise au piège de l'ogresse. Cette fois-ci, les excréments du héros, laissés sur les branches en haut d'un arbre, distraient l'ogresse par des rires en lui faisant croire que les enfants ours sont montés dans l'arbre.

Kwálá aġa naXəlčə'maq,

šá :Xlix wámqu,

IXk’ayáwəla. 
Gagyuxánawənx, gagyúlxam,

«Ána :' : !

« Dánba imíwkwł -

« imímxix šáXlix?

«Ayulkwčwáya,

K’úya.

« Í zukX ! "

Bientôt alors, elle les entendit,

haut dans l'arbre,

ils riaient.

Elle regarda là-haut,

Elle leur dit,

"Oh la la!

Pourquoi tu l'as amené en haut -

ton frère, là-haut ?

Il pourrait tomber,

Rien.

Descend-le!»

Les excréments-appâts finissent par lui tomber sur la tête déterminant ainsi un changement radical dans le rapport entre Wasguk'mayli et Kitsimani. En effet, piégée pour la troisième fois, cette dernière meurt ${ }^{4}$. Issu d'une série de trois événements similaires, créant un parallèle et configurant logiquement les trois actes, cet acte (I) termine la première sous-partie.

La coupure entre ces trois actes et les suivants est également marquée par l'emploi renforcé des marqueurs rhétoriques du quatrième :

Yaxa láyč gałúya,

kwálá łúyt, ag̉a gałilúłXamix. ${ }^{5}$

Nous remarquons aussi dans cette nouvelle séquence d'actes le changement de contexte du premier $(\mathrm{J})$ où les frères ours sont sauvés puis hébergés et nourris par Grue, personnage paternaliste chez les Chinooks. Fin de la fuite. Leur aventure est relatée à leur hôte par Wasguk'mayli. L'acte suivant revient sur Kitsimani qui mange le poisson séché empoisonné, laissé en appât par Wasguk'mayli, et "meurt » à nouveau. Cette victoire du héros, marquée par la dernière prise aux pièges fabriqués par lui, sert à inciter l'attente chez l'auditeur/lecteur d'une ogresse contrariée, à confirmer l'effïcacité et donc la sécurité des orphelins. Or tous les événements qui auront lieu après l'arrivée de Wasguk'mayli et de ses frères chez Grue seront lus dans ce sens.

Dans l'acte suivant, par exemple, la relation entre Kitsimani et Wasguk'mayli se rompt définitivement, malgré la persévérance de l'ogresse qui fait de son mieux pour franchir la rivière. Une scène comique se produit entre Grue (génie de la 
chute fatale) et l'ogresse qui part à la dérive. Pour les orphelins, c'est la fin d'un type de cohabitation et le début d'un autre. L'agresseur est écarté du lieu protégé, par un acte volontaire (le cinquième de ce style), mais aussi par la force naturelle de la rivière. Les deux sous-parties se combinent dans l'affaiblissement et l'achèvement réel de Kitsimani. Le dernier acte ajoute la touche finale du réconfort que Grue offre aux enfants: Wasguk'mayli lui demande si Kitsimani reviendra à nouveau et Grue lui répond aussitôt que non. Ainsi la prise de conscience des jeunes de leur nouvelle situation devient un facteur déterminant dans l'interprétation des événements.

Constituée de seulement trois actes, la troisième partie est consacrée au nouveau statut de Kitsimani par rapport à son environnement. L'acte $\mathrm{N}$ présente l'ogresse prisonnière d'un barrage et agressée par des corbeaux. Revenant à elle, elle chasse les oiseaux et se maquille le visage avec le sang des blessures occasionnées par les coups de bec à sa vulve, décrit par le terme clackamas utilisé pour le sang menstruel.

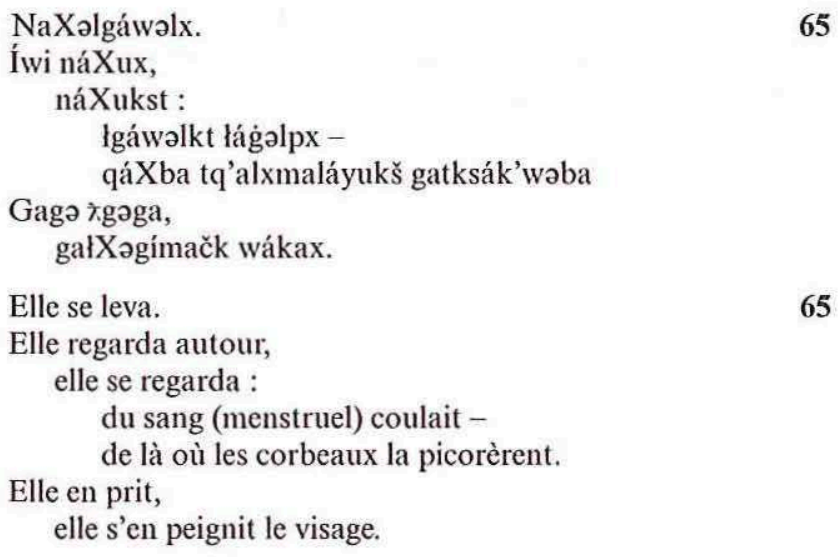

Cette nouvelle image de femme est mise à l'épreuve dans l'acte $\mathrm{O}:$ l'ogresse accorde des propriétés aux arbres, en les bénissant ou les condamnant en fonction des réponses bonnes ou mauvaises qu'ils donnent à propos de son image; elle annonce l'arrivée des humains dans ce nouvel ordre du monde. Dans la narration originale, Howard décrit les rencontres entre Kitsimani et quatre arbres. Elle raconte huit autres rencontres dans une narration transcrite et insérée par la suite. Ces rencontres se configurent selon une logique binaire et non tripartite selon le regroupement de vers identifiés. Ces vers s'organisent autour de quatre étapes, répétées presque à l'identique chaque fois :

L'arrivée de Kitsimani devant un arbre : face à face des deux entités

Kitsimani demande l'opinion de l'arbre 
L'arbre répond

Kitsimani accorde des propriétés à l'arbre.

Ces quatre vers créent une relation binaire d'interpellation/réponse par laquelle la réponse détermine systématiquement la formule incantatoire prononcée par l'ogresse à la fin de chaque rencontre. Ce changement de rythme en mesure de quatre, voire de deux fois deux, à la place des mesures de trois et cinq, reflète également le changement d'ordre dans le monde décrit et le nouveau rapport instauré par Kitsimani avec son environnement.

La versification binaire de la troisième partie s'impose aussi à un autre niveau. Le dernier acte $(\mathrm{P})$, qui constitue une scène et une intrigue bien différenciées des autres versions de cette histoire, tient plutôt lieu d'épilogue. Le changement abrupt du contexte et du comportement de l'acteur principal marque, en effet, une coupure nette entre l'acte P et les deux actes précédents, sans coïncider avec un résultat d'une séquence de trois. De plus, l'organisation binaire est maintenue dans le dernier acte, exprimé en quatre strophes de deux vers chacune. Cet acte met en scène l'arrivée de Kitsimani chez des gens du «Temps Moderne ». On raconte une version différente de l'histoire que nous venons d'entendre/lire. Dans ce nouveau récit, il est dit que Kitsimani mange tout le monde. Kitsimani répète stupidement cette version de l'histoire et s'y attarde.

Kwálá úyt, nugáqwam itġwa'li.

Náškupq, núlayt.

UXáxikwłila idə'lxam, ugwakíXaX, ugwagímnil, «Qínihú :ya --

Wasgukmáyli iłyámxikš -qúšdyaxa a :' : :nġa

Ảga ikłú 丸xum Wakitsimani. »

ÁXkamaX alagíma, « Kiniłúyakiniłúya. qúšdyaxa a :' : :nġa, gakłú ¡xum Wakitsimani. »

Kwaba :' : náXuX.

Káwux aga fúXwan qá gadə'kuX.

Ag̀a k'aník'ani.

Bientôt alors qu'elle s'en alla, $72 \mathrm{a}$ elle arriva à une maison. 
Elle entra,

elle s'assit.

Les gens parlent d'elle,

$72 b$

ils le font,

ils sont en train de dire,

"Quand ils s'en allaient --

Wasgukmáyli et ses petits frères --

c'était il y a longtemps,

Alors Wakitsimani les mangea. »

Alors elle disait aussi,

"Quand ils s'en allaient quand ils s'en allaient, ${ }^{6}$

c'était il y a longtemps,

Wakitsimani les a tous mangés. »

Juste là, elle resta. ${ }^{7}$

Le lendemain, alors je ne sais pas ce qu'il lui fit.

Alors voilà ce dont je me souviens.

Alors mythe mythe.

Howard termine ainsi sa narration de Waskíntwa kwaliwi Wakitsimáni išdáquq avec la formule clackamas courante de fin de mythe, $k^{\prime}$ ani $k^{\prime}$ ani (mythe mythe).

Il est évident que l'analyse de versification seule ne peut avoir d'intérêt que dans l'interprétation phonologique ayant une ambition prosodique de récitation orale. Ce qui n'est pas le cas ici, notamment parce que la phonologie et la prosodie chinook demeurent malheureusement inexplorées. Le schéma proposé ci-dessus nous guide uniquement dans la recherche de logique narrative et donc de sens. Or; c'est la recherche stylistique et narratologique qui permet l'analyse de la versification. L'approche ethnopoétique du récit oral trouve sa valeur dans une complétude entre l'organisation versifiée et d'autres signes formels.

\section{LOGIQUE NARRATIVE}

La narratologie structurale a depuis longtemps mis en évidence l'importance, pour la configuration narrative, des catégories temporelles qui s'expriment par la grammaire, l'ordre et la juxtaposition des événements, la durée de chaque événement, les rapports cosmologiques et saisonniers, les repères historiques. Nous avons dit que Waskíntwa kwalíwi Wakitsimáni išdáquq est construit sur des séries d'événements en séquences de trois et cinq et, vers la fin de la narration, sur des regroupements binaires ; nous avons constaté que ces événements sont présentés, pour la plupart, par ordre chronologique. Cette organisation temporelle du récit n'est néanmoins pas aussi simple qu'il y paraît. Interviennent dans la configuration trois matrices signifiantes, caractéristiques, pour les deux premières, de toute 
œuvre littéraire et, pour la troisième, de la poésie orale. La première, qui est identifiée et démontrée par Kenneth Burke (1931; Hymes 1987), rend compte de la façon dont le conteur cherche à éveiller et satisfaire les attentes de l'auditeur/lecteur. La seconde matrice concerne le phénomène du parallélisme en tant que caractéristique de la poésie, mis en lumière par le poète Gerard Manley Hopkins, systématisé en linguistique et en critique littéraire par Roman Jakobson. Enfin, notre étude sur la répétition (d'un acte, d'un motif, d'un dispositif, etc.) fait apparaître que, malgré des similitudes parfois redondantes entre les configurations, l'occurrence répétée crée toujours un nouveau sens ${ }^{8}$. Ces trois modèles, qui n'épuisent certainement pas la structuration du récit chinook, se décèlent à plusieurs niveaux du texte: dans son organisation temporelle, par exemple dans la durée narrative ou l'ordre des événements présentés ; dans les points de vue créés ; dans le placement des petites unités, par exemple des ellipses, des marqueurs rhétoriques, des tournures de parole ; et dans les divers plans de la métadiégèse ${ }^{9}$, l'occurrence d'un récit au sein d'un récit, ce qui aura une grande importance pour notre interprétation de Waskíntwa kwaliwi Wakitsimáni išdáquq. Regardons donc de plus près la composition du texte.

Avant même la première sortie des deux mères pour la cueillette des mûres, nous identifions un déséquilibre dans une situation domestique qui est peinte délibérément comme étant équilibrée et harmonieuse. Le préfixe nominal $\mathrm{Wa}$, qui figure dans le titre du texte aux noms des deux personnages maternels, disparaît du nom de Skíntwa ${ }^{10}$, alors qu'il est conservé dans la nominalisation de sa sœur. L'emploi de ce préfixe par Victoria Howard, qui est étudié par Hymes (1981, pp. 342-381), nous révèle la qualité expressive d'un tel détail :

In sum, the choice of wa- or a-is not random, nor is it to be explained within the immediate
sentence. The choice involves an active or passive state of the named actor, but the active
or passive, transitive or intransitive, causative or stative character of the accompanying
verb construction or verb stem is not a criterion in itself. The state of the named actor
depends upon the use of the sentence in the action of the story, an action that is shaped and
expressed through the rhetorical-poetic organization of the discourse. (ibid., p. 353)

Sachant que Kitsimani est l'ogresse la plus connue de la mythologie chinook, il n'est pas étonnant que ce préfixe soit attaché à son nom. Il est cependant important de remarquer que ce signe est en parfaite contradiction avec la configuration stylistique équilibrée du prologue et avec son contenu qui masque tout danger et désaccord. Ce préfixe nous fait ainsi un clin d'œil suggérant une légère ironie de la part de la conteuse ${ }^{11}$.

À la fin du premier acte (B) de la partie I, nous avons assisté aux sorties des deux sœurs pour la cueillette. La prédominance dans la littérature chinook de l'organisation numérique en trois et cinq provoque à elle seule des attentes chez l'auditeur/lecteur imprégné de cette culture. Cette composition rythmée accorde aux deuxième et quatrième battements le rôle d'une « note sensible » ${ }^{12}$, incitant 
l'anticipation d'une manifestation quelconque. Lors de la première sortie, de nombreux signes sont placés, indiquant un danger imminent (façon négligente de la part de Kitsimani de cueillir les mûres, sa présence physique imposante, etc.) et augmentant ainsi le suspense. La version « rideaux tirés » de la deuxième sortie fournit une ellipse qui laisse place à l'imagination de l'auditeur/lecteur dans la construction du récit. Il est important de comprendre que cette répétition ne fait pas simplement écho à l'événement mais qu'elle nous amène également à induire une troisième sortie dont la pertinence sera culminante. Cette culmination s'achève non pas par l'acte dangereux mais par l'avertissement que fait Skíntwa à son fils aîné du danger et de la nécessité de se préparer à y réagir. La description de la troisième sortie - et la conteuse indique clairement qu'il s'agit de la troisième tout au début de l'acte - est encadrée, nous l'avons vu, par deux types d'instructions données par la mère à son fils.

La répétition de l'avertissement de la mère ourse à la fin de l'acte $\mathrm{C}$ nous offre des élaborations signifiantes par rapport à celui du début de l'acte :

Ag̉a gasdúya,

gasdə'xk'wa gašdúyam.

Xábixix ag̉a naXaylgwə'ličk, ičáxan iXg̀'qwnq.

Ġagyúlxam ag̉a,

« Dáyax wígwa qámaX iga'nuX

Ayábadix iłmímxikš.

SámniX aladímama áyma, agəm ‡xáma,

"Kwábá ináqi đ̇q wámšaq.

Káwux anugálmama."

Qánaġa láxlax aga'mšitX.

Ag̉a agəndwágwaXda. "

Alors elles s'en allèrent,

elles s'en allèrent à la maison,

elles arrivèrent.

Le soir, alors elle l'informa, son fils qui fut l'aîné.

Elle lui dit alors,

«Aujourd'hui elle me fit dans tous les sens.

Fais attention à tes petits frères.

Si elle revient seule,

elle te dira,

"Là bas, j'ai laissé ta mère.

Demain, j'irai la chercher."

Elle essayera simplement de te duper.

Alors elle m'aura tuée. » 
Ce deuxième avertissement, structuré en trois vers constituant une strophe, est exprimé par un développement discursif de la locutrice représenté, à première vue, par le doublement du nombre de lignes récitées. Ce discours maternel fait d'abord un bilan de la menace subie par Skíntwa, ce qui crée un sentiment d'urgence. À l'opposé du premier avertissement, la mère commence ses instructions par " fais attention à tes petits frères » en ajoutant le suffixe diminutif $-\check{s}$ pour insister sur la petitesse (i.e. vulnérabilité) de ces derniers. De la même façon que la mère met l'accent sur ce qui vient de se passer, elle donne des détails sur ce qui va se passer en précisant les propos que tiendra Kitsimani quand elle rentrera seule de la cueillette. Ces « ajouts » sont complétés par plusieurs opérations de langue à l'intérieur de cette strophe. En premier lieu, les dires de Skíntwa sont introduits par deux marqueurs de discours. Dans le premier avertissement, le narrateur nous indique, "alors elle dit à l'aîné [de ses] son fils 》. Dans le deuxième, nous lisons : « le soir, alors, elle l'informa de son expérience,I son fils [qui était] l'aîné »; et plus loin : « elle lui dit alors [...] ». Par rapport à la première formule, Howard invertit l'adjectif qualificatif "aîné " et son sujet dans

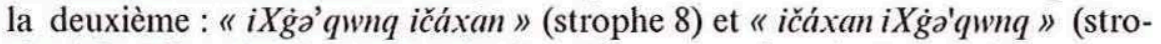
phe 16). Elle ralentit encore plus le rythme avec deux instances du marqueur rhétorique $a \dot{g} a$ (" alors »). Le verbe-IXam, qui est le marqueur de discours le plus commun dans les textes chinook et qui indique, en plus d'un acte verbal, une interpellation, n'est utilisé que dans un deuxième temps ${ }^{13}$. Howard emploie, dans un premier temps, la racine verbale-gw(a)' li qui signifie « informer » et aussi une révélation, ce qui insiste plus sur la transmission d'une information. De ce fait, ce vers n'est pas une simple préface. L'élément réflexif - $X a$ - nous indique, de manière très laconique, que l'information transmise porte sur la locutrice. La conteuse ajoute à ce complexe verbal le suffixe-čk, aspect perfectif qui donne ici le sens d'un accomplissement formel à l'énonciation de la mère ou du devoir d'une autorité ou encore de ses dernières paroles. L'effet dramatique de ces opérations colore une scène émouvante dans laquelle nous découvrons la tendresse d'une mère pragmatique et déterminée et dans laquelle l'on peut espérer une prise de conscience de la part du fils.

Nous avons affaire dans cette strophe à deux métadiégèses qui contiennent une répétition laconique de la narration précédente et de la suivante. Le marqueur " sámnix » dans la ligne « si elle revient seule » exprime une hypothèse, ce qui dénote chez Skíntwa un léger espoir de ne pas voir les événements décrits se produire. Néanmoins, d'un point de vue strictement textuel, la métadiégèse fera du meurtre une répétition. Sa fonction est donc de développer le suspense, mais aussi de mettre en rapport direct l'acte du meurtre et l'interlocuteur qui ne sera ni acteur ni observateur de cet événement. L'embrayage du point de vue, que rend possible la forme métadiégétique, établit un lien implicite entre Wasguk'mayli et Kitsimani. Entre alors sur l'avant-scène le fils aîné de Skíntwa, mais de manière fort discrète jusqu'à la cinquième et dernière sortie pour la cueillette. Le person- 
nage n'apparaît pas dans les événements de l'acte $\mathrm{D}$ où a lieu le massacre de sa mère ; cependant, il reste présent dans l'esprit de l'auditeur/lecteur qui, tout comme lui, aura attendu le meurtre. La mise en relation de l'ogresse avec Wasguk'mayli est signalée par la répétition métadiégétique faite par le fils quand Kitsimani rentre de la cueillette sans sa mère : "Alors elle l'a tuée ».

C'est donc l'inéluctabilité du meurtre tragique et la prévision des nouveaux dangers qui sont mis au premier plan en tant que nouvelle information (ou rhème), alors que la rupture elle-même est régie comme un thème donné. Notre attention est portée, non pas vers l'acte violent qui aura lieu dans le troisième acte, mais vers les interactions entre l'ogresse et son entourage social. C'est effectivement le comportement de l'ogresse et la réponse des autres à ses gestes et à ses dires qui sont décrits au plus près dans ces trois actes.

La culmination de trois actes conséquents dans le deuxième de la séquence formelle n'est pas sans intérêt. Elle crée un rythme syncopé dans la relation des événements, nous propulsant de manière insidieuse en plein milieu de la scène tragique. La sœur féroce exerce dans cette scène une force verbale, qui affaiblit sa proie ${ }^{14}$, et une force physique dans un acte tragico-comique de goûter, de meurtre et de mutilation corporelle à des fins culinaires. Nous sommes ici témoins d'une relation dominante/dominée, mais avec un doigt pointé sur le pouvoir maternel de la dominée, qui a déjà été transmis à son fils. En plus de la répétition métadiégétique, l'importance de cette transmission est exprimée, comme Hymes (1981, p. 346) nous le fait remarquer, dans la nouvelle occurrence du préfixe $W a$ attaché au nom de la mère ourse dans l'acte $\mathrm{E}$, nominalisation de puissance faite à l'intérieur du récit uniquement par rapport à ses enfants. Nous lisons : « les enfants de Waskíntwa ».

Nous identifions plusieurs parallèles dans les actes C et E qui renforcent le lien triangulaire entre Skíntwa, Wasguk'mayli et Kitsimani. Ces deux actes servent de $<$ déroulement $>$ dans l'organisation des unités formelles. Ce qui est bien plus frappant, c'est que, placés entre des actes plus axés sur l'action, $\mathrm{C}$ et $\mathrm{E}$ donnent lieu à la réflexion et à la prise de conscience de la situation changeante, alors que $\mathrm{B}, \mathrm{D}$ et $\mathrm{F}$ relatent des actions continues. Au niveau de la structure, l'acte E, comme le C, est encadré par des événements semblables. Il s'agit dans un premier temps du retour de Kitsimani (sans la mère ourse) et, le lendemain, de son départ pour la cueillette. Mais la similitude ne se réduit pas à ce « retour/départ » d'une mère. Ces deux événements se complètent aussi par la mise en relation d'une manière d'abord implicite (acte C) et puis explicite (acte E) de Kitsimani avec Wasguk'mayli.

La conteuse emploie plusieurs dispositifs pour mettre en relief la lutte de pouvoir complexe qui se développe entre l'ogresse et les orphelins. Dès le retour de l'ogresse, le point de vue narratif se dirige vers son nouvel opposant sur le plan psychologique : "Alors il pensa : "Alors elle l'a tuée" ». Cette première strophe nous précise ensuite: «Rien du tout lui dit-il» (ce qui sera répété dans les 
deuxième et dernière strophes de cet acte). Motif récurrent dans les œuvres littéraires - le dominé se tait devant le dominant -, ce mutisme est accentué, dans notre récit, par les pleurs du bébé dans la nuit qui sent rôtir les seins de sa mère, gourmandise pour l'ogresse. Restant silencieux, Wasguk'mayli détourne les mensonges de l'ogresse qui veut lui faire croire que tout est de l'ordre du rêve et que, le lendemain, elle partira chercher la mère ourse. Ce mutisme stratégique donne également un temps de réflexion à l'auditeur/lecteur qui, témoin de l'atroce effet produit par la mort d'une mère sur son bébé, anticipe la réaction de l'aîné qui devient responsable de ses jeunes frères.

Une relation dialectique de mystification se crée ici : la non-vérité des dires de la dominante perd sa valeur mystifiante dans la manipulation de la vérité à travers le mutisme du dominé. Cette contradiction est synthétisée par les cris du bébé en tant qu'acte communicatif instinctif dont le contenu est hors de portée du vérifiable. Nous remarquons aussi que le mutisme de l'aîné est à chaque prise une réponse aux actes et dires mensongers de l'ogresse, alors que les cris du bébé servent d'accusations auxquelles l'ogresse réplique par des mensonges, ce qui la rend subordonnée à la communication du plus faible des trois. Nous trouvons, sur ces deux plans, une inversion du pouvoir dans les actes communicatifs qui bouleversent, quoique provisoirement et de manière tacite, le statu quo du domicile.

L'anticipation du renversement des rôles dominé/dominant est comblée par le dernier acte (F) qui se termine par le départ des cinq ours, le plus petit sur le dos de l'aîné. Ce dernier est en plein calcul et activité alors qu'il construit quatre pièges (chiffre sensible) dans le but de ralentir l'ogresse. Le pouvoir inhibé chez la mère transmis à son fils dans l'acte $\mathrm{C}$ maintient cette inhibition dans l'acte $\mathrm{E}$ mais, dans l'acte final de la partie I, ce pouvoir trouve sa manifestation dans la naissance d'un héros plein d'énergie et de ressources imaginaires. Les attentes de l'auditeur/lecteur sont satisfaites. Nous avons esquissé ici un certain nombre de dispositifs qui montrent que la première partie de notre récit est composée selon une logique événementielle et rhétorique. Par manque de place, nous avons bien évidemment laissé de côté maints autres signes stylistiques figurant dans la configuration du texte. Afin de revenir à une comparaison entre les approches de Hymes et de Lévi-Strauss, il est à présent nécessaire de montrer les relations existant entre les trois parties qui permettent l'émergence de la sémantique narrative propre au texte de Victoria Howard.

\section{Structures mÉtadiÉgÉtiQues}

Nous rencontrons dans Waskintwa kwaliwi Wakitsimáni išdáquq vingt-neuf occurrences de métadiégèses dont seize se trouvent dans les deux premières parties : huit dans la partie I et huit dans la partie II. Onze de ces seize récits 
reflètent un événement présenté par le récit premier, cinq portant sur l'avenir (prolepse) et six sur le passé (analepse). Trois autres récits contiennent les histoires mensongères de l'ogresse qui relate tous les événements passés dans le but de déterminer ceux du futur. Deux des quatre récits dits par Grue portent sur l'avenir sans refléter des événements décrits par le récit premier. Les treize rencontres entre Kitsimani et les arbres dans la partie III donnent lieu à treize métadiégèses de nature proleptique : « Les gens arrivent bientôt [...] ». Les deux derniers récits insérés dans Waskíntwa kwalíwi Wakitsimáni išdáquq, nous le verrons plus loin, portent sur l'instance narrative même, autrement dit sur la narration que fait Howard pour Jacobs et pour leurs lecteurs. Nous examinerons tout d'abord les seize récits tissés tout au long des parties I et II.

Comme pour toute métadiégèse, celles de notre récit privilégient les points de vue de leurs narrateurs personnifiés. Ces structures nous dévoilent ainsi l'importance, dans Waskintwa kwalíwi Wakitsimáni išdáquq, du point de vue de Wasguk'mayli qui est l'auteur de quatre récits et qui assiste à la configuration d'un cinquième, et aussi celui de Kitsimani qui en récite dix-neuf. Skíntwa est la source des trois premières métadiégèses, Grue des trois dernières - dans le troisième, il est co-narrateur avec Wasguk'mayli. Notons cependant qu'il ne suffit pas qu'un personnage raconte une histoire pour créer une relation hiérarchique de son point de vue. La narration elle-même doit être considérée par rapport au déroulement du récit premier, aux relations interpersonnelles et aux opérations discursives. Nous remarquons par exemple que, malgré l'importance du nombre de récits configurés par Wasguk'mayli, leurs formes performatives sont faibles. En effet, seul l'un d'entre eux est auditionné par un public propre. Le tout premier récit, nous le rappelons, prend la forme d'une réflexion silencieuse de la part du héros et, du coup, fait écho aux dires de sa mère. Les deux suivants constituent de véritables mises en scènes avec, premièrement, le chien et, ensuite, les excréments du héros. Il s'agit là d'êtres animés, certes, mais sans les facultés de personnages autonomes. Ce n'est qu'à son arrivée chez Grue que Wasguk'mayli aura un public anthropomorphe. À la fin de l'acte J, nous lisons : « Il lui raconte/révèle tout ». II s'agit plutôt d'une méta-métadiégèse car il ne dévoile que le fait de relater, laissant en suspens toute trace d'interprétation des événements. Cependant, l'effet de sa narration sera conséquent, puisqu'il a une force perlocutionnaire, alors que Grue s'investira définitivement dans la résolution du conflit.

Les deux métadiégèses proleptiques de Skíntwa servent aussi de mise en scène. Cependant, elles sont auditionnées par un acteur « humain » qui agira en fonction des événements qu'elle raconte. Interprétation perlocutionnaire qui influera sur la suite des événements, son récit analeptique, comme nous l'avons vu, a la fonction d'informer (d'ouvrir les yeux de) son interlocuteur en relatant ce qui va lui arriver. Chez Grue, nous avons aussi affaire à deux récits de nature perlocutionnaire. Ce personnage relate d'abord à Kitsimani que c'est en traversant la rivière sur sa jambe que les enfants sont arrivés de l'autre côté. Il met ainsi 
l'ogresse au défi de tenter la traversée qui la mènera à sa chute finale. Ensuite il raconte cette chute aux enfants afin de calmer leurs inquiétudes. Quand Wasguk'mayli évoque la possibilité du retour de Kitsimani, Grue met fin aussitôt à un tel scénario avec un simple " non », ce qui clôt cette dernière métadiégèse ainsi que l'aventure des fuyards et la deuxième partie du récit premier. Dans ces deux cas, nous identifions le premier type de métadiégèse décrit par Gérard Genette : celle qui permet « une causalité directe entre les événements de la métadiégèse et ceux de la diégèse », mais il ne s'agit pas, comme dans les exemples fournis par Genette (1972), d'une forme qui « confère au récit une fonction explicative ». Il s'agit d'une relation d'événements qui déterminera les actes de son (ou de ses) récepteur(s) plutôt qu'elle ne tentera de clarifier ou d'expliquer les événements pour l'auditeur/lecteur.

Un changement important dans le fonctionnement des métadiégèses s'impose dans la partie III. L'ordonnancement des propriétés aux arbres que prononce Kitsimani dans une forme métadiégétique crée une équation entre métadiégèse et diégèse, alors que l'acte verbal constitue l'acte narré qu'il décrit. Il n'est pas nécessaire que le récit premier nous précise que l'ordonnancement reflète les propriétés véritables des arbres connus et exploités plus tard par les Chinooks. En tant que verbalisation cérémonielle, nous avons affaire à un acte illocutoire, mais de l'ordre imaginaire (mythique) et non administratif. Inséré dans le récit premier, l'ordonnancement établit une véritable fusion avec la diégèse en tant qu'acte déterminant bien au-delà d'une locution.

Les récits insérés au sein de Waskintwa kwaliwi Wakitsimáni išdáquq servent donc plusieurs fonctions. Ils rythment le texte tout en variant les points de vue sur les événements et en conjuguant le présent de l'histoire avec son passé et son futur. En tant qu'échanges communicatifs, ils établissent des liens interpersonnels entre les divers personnages. Nous remarquons alors que les récits de Wasguk'mayli marquent une évolution sociale du jeune personnage. La force perlocutoire du récit de Skíntwa se traduit par la remarque laconique du fils, «Alors elle l'a tuée », qui condense les instructions élaborées par la mère. Cette réflexion marque le début de l'autonomie du jeune grâce aux précautions maternelles. Des actes diégétiques limités le conduisent à maîtriser ses ressources (quoique limitées) avant de trouver l'aide ultime chez Grue. Et c'est à cet être bienveillant que Wasguk'mayli relate ses aventures, créant ainsi un lien entre les actes d'interprétation et ceux d'intégration sociale. Ce récit non détaillé couvre jusque-là tous les événements de l'histoire et renvoie ainsi l'auditeur/lecteur à une nouvelle interprétation. Ces procédés métadiégétiques révèlent l'importance dans ce récit de la transmission des informations d'une mère à son fils, mais aussi de la capacité à interpréter ces informations. Un survol de l'histoire qui nous est racontée se résume alors trois fois par des métadiégèses : dans le récit initial de Skíntwa, dans le récit métonymique de Wasguk'mayli et dans celui que ce dernier fait à Grue. 
Il serait utile de faire une pause ici afin de regarder la sémantique narrative de ce récit. Si nous nous fions bien aux signes et aux modèles du récit de Victoria Howard, un ensemble de thèmes émergent nous permettant de constater l'interprétation particulièrement féminine que Victoria Howard fait de ce mythe. Nous identifions tout d'abord l'importance du pouvoir créateur et destructeur del'acteur principal sur le déroulement narratif. Kitsimani tue sa sœur, détruisant ainsi le foyer cohabité, et menace violemment la sécurité de ses neveux. Cependant, par un jeu créatif d'identité sexuelle féminine, elle devient ordonnatrice de propriété naturelle, afin que la nouvelle société puisse se développer. Le pouvoir féminin se manifeste pour la première fois en tant que thème principal à travers le personnage de Skíntwa. Le rôle de cette force qui s'oppose au personnage principal dévoile l'importance de la transmission du savoir et du pouvoir entre les générations, non seulement pour la survie mais aussi pour le développement d'un esprit prévisionnel et pragmatique afin de surmonter les tragédies inévitables. Victoria Howard met en avant la force protectrice de la mère et la force créatrice de la sexualité féminine.

En plus de ces accentuations féminines, Victoria Howard exécute, dans son récit, diverses opérations de mystification et de démystification des pouvoirs sociaux, personnels, matériels et spirituels. Un certain nombre d'actes de mystification - tromperie, appâts, mensonges, mutisme, maquillage féminin, etc. - est décrit non pas comme des manœuvres mécaniques, mais de manière à ce que les relations entre les personnages se lisent à travers eux. Les opérations d'illusion, qui déterminent le déclenchement de l'histoire et sa tonalité et qui maintiennent une tension permanente entre les forces créatrices (transmission générationnelle, fabrication matérielle, sexualité féminine, ordonnancement) et destructrices (violence physique) servent également de trope littéraire, alors que le personnage principal mythique se transporte dans le « Temps Moderne » à la fin de l'histoire et y devient conteuse.

Afin de mieux cerner l'importance métaphorique de la configuration narrative de ce texte, nous regarderons maintenant de plus près les métadiégèses dites par Kitsimani. Nous avons vu que les premiers récits de l'ogresse consistent en des mensonges qui n'aboutissent pas à leurs fins, ce qui démontre le caractère débile du personnage. Ensuite Kitsimani compose des diégèses proleptiques pour accorder aux arbres leurs valeurs dans l'économie humaine à venir. De menteuse à démiurge, Kitsimani a un rôle qui évolue considérablement. Nous arrivons maintenant à la fin de notre récit où ressurgit l'état plutôt passif de Kitsimani et où elle semble avoir perdu son pouvoir. Nous reproduisons ici cet extrait pour faciliter la lecture :

Bientôt alors qu'elle s'en allait, elle arriva à une maison.

Elle entra, elle s'assit. 
Les gens parlent d'elle,

$72 b$

ils le font,

ils sont en train de dire,

"Quand ils s'en allaient -

Wasgukmáyli et ses petits frères -

c'était il y a longtemps,

Alors elle disait aussi,

Alors Wakitsimani les mangea. »

"Quand ils s'en allaient quand ils s'en allaient,

c'était il y a longtemps,

Wakitsimani les a tous mangés. ”

Juste là, elle resta.

$72 \mathrm{c}$

Le lendemain, alors je ne sais pas ce qu'il lui fit.

Alors voilà ce dont je me souviens.

Alors mythe mythe.

En tant que métaphores de l'histoire qui vient d'être racontée, les deux dernières métadiégèses nous renvoient à nouveau à l'interprétation des événements tout en se trompant sur la fin. Mais ce procédé ne s'arrête pas là. La « mauvaise » version racontée par les humains est en effet répétée par l'acteur principal du récit premier. Soulignons que, dans ces deux derniers récits, Kitsimani est à la fois personnage principal et public, et qu'ensuite elle en devient la conteuse $^{15}$. Ces métadiégèses condensent ainsi tous les éléments essentiels à la configuration narrative : le narrateur, le narrataire, les événements, le personnage et l'acte d'interprétation. De plus, elles mettent en exergue la relation entre les événements de l'histoire et ceux du récit ainsi que la narration elle-même - les trois aspects de la réalité narrative telle qu'elle est décrite par Gérard Genette. Il ne fait aucun doute que, dans cet acte épilogique de Waskíntwa kwaliwi Wakitsimáni išdáquq, Victoria Howard attire notre attention sur l'instance même de sa narration et sur des questions d'interprétation. La répétition stupide, dans un langage mal formulé par Kitsimani, nous amène à croire que cette toute dernière métadiégèse est de l'ordre de la simple locution. Cependant, un nouveau regard sur les modèles, les signes et les thèmes soigneusement manipulés dans le récit premier; invite à une interprétation plus profonde. Cette dernière métadiégèse porte-t-elle sur le passé ou sur le futur? La conteuse, serait-elle en train de mentir ou d'ordonner au lieu d'imiter ? Est-ce que cette répétition cache un mutisme de la part du personnage principal ? A-t-elle vraiment perdu son pouvoir d'ogresse? "Peut-être sera-t-elle encore de retour ? ", nous demandons-nous, à l'instar de Wasguk'mayli, en revenant sur notre démarche narrative.

La fonction performative du récit de Kitsimani est, dans tous les cas, ambiguë entraînant donc plusieurs sens. Une analyse ethnopoétique exige avant tout une explication cohérente par rapport à l'isomorphisme du texte. En prenant en 
compte toutes les implications du pouvoir métaphorique de ces deux dernières métadiégèses, Victoria Howard nous fait aisément comprendre que son propre récit propose une interprétation. Parallèlement, elle met en suspens l'avenir de cette interprétation. Au départ de l'histoire, là où Victoria Howard a composé des vers symétriques reflétant une situation dangereuse mystifiée dont le seul signe explicite du danger, rappelons-le, est l'emploi du préfixe wa-, cette composition stylistique et l'élément contrastant nous indiquent que Victoria Howard avait, dès le début, l'intention de fournir à son interlocuteur un récit soigneusement configuré. Elle aura cherché tout le long de sa récitation à le renvoyer (et nous avec lui) sur ses pas afin de décoder les signes de la configuration.

Dernier retour sur l'interprétation du récit de la conteuse : cette dernière métadiégèse énoncée par Kitsimani ajoute un aspect métaphysique spécifique à la récitation de Howard; elle s'appuie sur le rôle ambigu de Kitsimani et, par extension, sur celui de Howard elle-même en tant que narratrice. Cette métalepse évoque de nombreuses hypothèses sur le point de vue narratif manipulé par l'auteur. Il est fort possible que Victoria Howard, croyant aux pouvoirs surnaturels des esprits tutélaires, doutait de la capacité du linguiste (et de leurs lecteurs), qui ne partageait pas ses croyances, à interpréter ce mythe. Cela expliquerait le développement de la forme métadiégétique qui incite l'auditeur/lecteur à revenir sur son interprétation. Textuellement parlant, la présence de Kitsimani dans l'événement narratif peut aussi s'expliquer par la synecdoque du monde mythique que représente l'ogresse.

Dans cette dernière optique, le déplacement du personnage mythique vers le « Temps Moderne » se reflète dans celui des Clackamas vers le monde occidental, à travers le passage du discours oral à l'écrit et de la langue clackamas à l'anglais. S'interrogeant lors de cette récitation sur des questions d'interprétation, Victoria Howard se demandait certainement quel était l'avenir de son récit et, par extension, de la langue et de la culture clackamas qui semblaient, déjà à ce moment, en train de s'éteindre. L'attention qu'elle a prêtée à la composition stylistique et symbolique de Waskintwa kwalíwi Wakitsimáni išdáquq indique clairement qu'elle maîtrisait bien le pouvoir de la mystification artistique et qu'elle espérait de son auditeur/lecteur une compétence d'interprétation symbolique.

INTERPRÉTATION MYYTHOGRAPHIQUE COMPARATIVE DE WASKÍNTWA KWALIWI WaKITSIMANI ISDAQUQ

Seule une lecture des Mythologiques permet d'apprécier la fine connaissance que Lévi-Strauss a du récit de Victoria Howard. Nous reprendrons ici les points qui nous semblent les plus féconds pour l'analyse ethnopoétique, mais aussi les plus représentatifs de l'analyse comparative faite par le mythographe. Selon Lévi-Strauss (1971, p. 254), le récit de Howard appartient au groupe de mythes 
« les faons et les oursons », relatant " l'avènement de l'humanité, rendu possible par l'instauration des foires et des marchés qui permettront aux humains de s'alimenter selon la culture : en échangeant leurs moyens de subsistance respectifs ». Dans ce récit, il est question de l'origine de l'échange commercial à son stade le plus fondamental, du développement de la cuisine et de la culture des moyens facilitant leur échange (feu, fumée, outils, taxinomie, etc.). « Il s'agit donc ici d'une création qui, dans l'univers zoologique et botanique, met l'accent sur des êtres dotés d'une valeur particulière aux yeux de peuples essentiellement pêcheurs, artisans du bois et vanniers » (ibid., p. 262).

Lévi-Strauss expose, dans sa lecture des mythes " les faons et les oursons », les rôles du désordre, de la cohabitation et de l'individuation dans l'organisation sociale. Il démontre que les variantes de ce groupe de mythes suivent plusieurs chemins selon un codage biologique, météorologique, astronomique, sociologique ou botanique (voir tableau, ibid., p. 261). Le mythe clackamas désigne dans cette analyse un codage biologique, notamment de la fabrication du feu. L'avènement de l'humanité provient d'une distinction entre la société et la nature, en rapport avec l'échange qui «n'a pas de place dans la nature où nul moyen terme n'existe entre le repliement sur soi et l'agression dirigée contre autrui » (ibid., p. 254). La socialisation réside dans ce « moyen terme " que l'auteur consacre aux origines matérielles et rituelles, telles que la cuisine, l'échange commercial, les tabous et les règles de bienséance à table qui font l'objet des Mythologiques.

Cette approche mythographique fournit des motifs et des formules utiles à une étude sur la cohabitation dans le texte de Howard. Écoutons Lévi-Strauss (ibid., p. 258) : « d'une façon générale, la cohabitation d'espèces animales aux mœurs incompatibles représente, dans le langage de la taxinomie, un scandale du même ordre que les autres couches mythologiques signifient au moyen de l'inceste des germains ». La séparation des espèces et de leurs nourritures est effectivement un thème développé dans le récit que nous fournit Howard. En remarquant, dans son étude structurale, le parallèle qui existe entre la cohabitation des deux familles d'ursidés et l'inceste des germains trouvés dans d'autres récits mythologiques de la côte nord-ouest américaine, Lévi-Strauss fait comprendre que la dégénérescence de la famille ourse noire/ourse grizzly ne doit pas être interprétée en tant qu'événement isolé, mais plutôt comme un stade dans le développement naturel et social du monde tel que les Clackamas le connaissaient et le vivaient. Il attire ainsi notre attention sur la relation du mythe à l'organisation sociale des cultures dont il est issu.

La structure de tous les mythes du groupe constitué, selon cette analyse anthropologique, s'organise autour d'une série de relations transformationnelles qui peuvent être décrites ainsi :

[...] qu'il s'agisse du soleil et de la lune ou de personnages qui les incarnent, de constellations en opposition de phase, d'alliés par mariage opposés au titre de donneur 
de femme ou de preneur, ou encore d'animaux relevant d'espèces antagonistes, un même schème commence par rapprocher des termes incompatibles. Cette proximité déclenche une crise d'où résulte une première disjonction marquée d'une valeur négative. Une seconde conjonction la neutralise. Poussée ou non jusqu'à son terme, elle laisse le champ libre à une nouvelle disjonction, marquée cette fois d'une valeur positive. L'étude exhaustive du groupe des faons et des oursons devrait tenir soigneusement compte d'un fait : cette seconde disjonction s'opère par l'intermédiaire du passeur susceptible jouant un rôle qu'on aimerait appeler semi-conducteur : il transporte les uns, intercepte les autres. (ibid., pp. 259-260)

Si nous prenons comme exemple le récit de Howard, la disjonction à valeur négative s'achève par la tuerie de Skíntwa et la fuite de ses enfants. L'arrivée des enfants de la victime chez Grue marque la conjonction qui neutralise la crise d'abandon que la fin de la cohabitation a créée pour la famille ourse. Le personnage paternaliste sert en effet de « semi-conducteur », alors qu'il « transporte » les enfants chez lui et « intercepte » l'ogresse, déterminant ainsi la disjonction à valeur positive. Cette structure tripartite correspond nettement aux trois parties de Waskíntwa kwalíwi Wakitsimáni išdáquq décrites par l'analyse de la versification, ce qui nous permet de constater une compatibilité des macrostructures identifiées par les deux méthodes. Nous verrons ci-dessous comment ce modèle s'encode dans le récit.

\section{CONCLUSION : CROISEMENTS ET CONVERGENCES}

Alors que l'interprétation comparative de Lévi-Strauss nous fournit un paradigme de valeur traditionnellement conçue (< disjonction - conjonction disjonction >) pour penser le sens de Waskíntwa kwalíwi Wakitsimáni išdáquq, les éléments et leurs relations dégagés par une telle démarche prennent une tout autre complexité lorsqu'on les examine à l'intérieur du texte. Nous remarquons, par exemple, des opérations dialectiques dans le lien spirituel, que représentent les mots de la mère dans les souvenirs du fils, et dans le lien sensuel, qu'expriment les pleurs du bébé alors qu'il sent rôtir les seins de sa mère, liens qui forment une sorte de conjonction issue de la mort (disjonction) de la mère. La complexité narrative de la conjonction/disjonction se trouve aussi dans les séquences qui amènent à la séparation finale de l'ogresse, dernière disjonction établie par sa chute finale. Après chaque prise au piège, Kitsimani revient à elle, toujours plus en colère. Elle se met aussitôt à chercher avec fureur les fils de Skíntwa. La séparation des espèces est décrite, dans ces épisodes, à travers des plans psychologique, social et spatial et peut être étudiée en valeur rhétorique dans le texte de Howard selon chaque plan. Finalement, cette dernière disjonction, qui éloigne l'ogresse du nouvel ordre social, la réintègre sur le plan métaphysique en tant qu'ordonnatrice, mais aussi sur le plan social lorsqu'elle s'approprie une puissance féminine 
constituant une force esthétique, sexuelle et créatrice. En partant ainsi de l'analyse comparative, l'analyse ethnopoétique de Waskintwa kwaliwi Wakitsimáni išdáquq nous amène à reconnaître une deuxième conjonction. Cette deuxième conjonction se confirme par l'épilogue du récit de Howard dans lequel Kitsimani s'immisce en tant que narratrice du mythe. L'étude du rapport cohabitation/noncohabitation, telle que nous l'avons développée dans le cadre d'une thèse de doctorat et qui a servi ici de point de départ, montre que nous avons affaire dans cet épilogue à une « différence significative » (Mason 1999).

Dans cet exemple d'échange entre les approches, les macrostructures du texte fournies par les deux analyses faites à part se ressemblent. C'est seulement dans l'application du modèle comparatif à la logique textuelle démontrée par la co-variation de la forme et du contenu (du plus large au plus petit) que nous dégageons une nouvelle dimension. Paradoxalement, la mythologie comparative peut porter notre attention sur un détail du texte qui n'est pas sous-jacent dans la lecture versifiée, mais qui peut être réinterprété à partir d'elle. Notre exemple consiste en l'empiétement dénaturé du territoire par des espèces différentes, point sur lequel Lévi-Strauss (1971, p. 254) insiste dans son analyse. Non seulement Skíntwa et Kitsimani partagent le même territoire, mais cette dernière, dans son but de dominer et, plus tard, de dévorer sa sœur, aide celle-ci à remplir son panier. L'approche physique de l'ogresse se déroule en trois étapes successives à partir du partage (dénaturé) du territoire.

La sœur aînée aide sa petite sœur.

Les deux sœurs se dépouillent, l'une l'autre.

La sœur aînée mange sa petite sœur.

Le partage du territoire est, tout comme le préfixe $\mathrm{Wa}$-inégalement distribué, un signe de danger, mettant en relief les questions de proximité et des mystifications féminines (aider l'autre - s'entraider - détruire l'autre). En effet, le partage - de foyer, de préoccupations maternelles, de canoë, de parcours et de ronciers'élabore dans le récit de Howard et s'achève, comme dans les autres versions du mythe, par la destruction. Encore une fois, la configuration harmonieuse, qui se traduit tant par la forme que par le contenu, contraste avec un phénomène qui exprime de manière implicite un «scandale».

Nous avons affaire, dans ces deux exemples, à des transpositions de structures identifiées par Lévi-Strauss aux analyses de la versification et qui viennent enrichir l'interprétation ethnopoétique fournie ci-dessus. À un tout autre niveau d'étude, les méthodologies lévi-straussienne et hymesienne se recoupent dans leur traitement, l'une de l'infrastructure des sociétés amérindiennes, l'autre plutôt de leur superstructure. Cherchant dans sa lecture des mythes toutes références à l'organisation et l'orientation sociales - phénomènes historiques, relations « institutionnelles », échanges économiques, modes de production - Lévi-Strauss 
induit les grandes étapes et conditions de sa fondation. De son côté, Hymes privilégie la négociation sémantique culturelle qu'entreprennent des individus face aux perplexités, aux difficultés et aux contradictions de la vie sociale alors qu'ils se réunissent et s'expriment dans l'arène de la performance. S'appuyant sur les codes de la communication, l'ethnopoétique étudie les idées, les valeurs et les compétences communicatives établies par les membres d'une société donnée dans sa confrontation à la vie réelle. Prises ensemble, ces deux méthodes réunissent les outils et les optiques nécessaires pour satisfaire cette exigence scientifique qui mérite rappel :

Les formations brumeuses du cerveau humain sont elles aussi des sublimés nécessaires du processus matériel de leur vie, empiriquement vérifiable et lié à des circonstances matérielles préalables. Par conséquent, la morale, la religion, la métaphysique et tout le reste de l'idéologie ainsi que les formes de conscience qui leur correspondent, ne conservent plus leur semblant d'indépendance. Elles n'ont ni histoire ni développement ; ce sont, au contraire, les hommes qui, en même temps qu'ils développent leur production et leur communication matérielles, transforment, avec cette réalité qui leur est propre, et leur pensée et les produits de celle-ci. Ce n'est pas la conscience qui détermine la vie, c'est la vie qui détermine la conscience. Dans la première conception, on part de la conscience comme individu vivant ; dans la seconde, qui correspond à la vie réelle, on part des individus eux-mêmes, réels et vivants, et l'on considère la conscience uniquement comme leur conscience. (Marx 1982, pp. 1056-1057).

Si l'enquête consiste à savoir comment vivaient les Amérindiens au plus lointain, à connaître la vie et la pensée précolombiennes, l'anthropologie structurale de Lévi-Strauss nous propose une démarche fructueuse et vaste dans ses applications. Mais si l'on s'interroge plus précisément sur les connaissances et les croyances transmises, voire transmissibles, par les narrations mythologiques, ou si l'on vise à réintégrer les dires d'un individu dans un système culturellement et historiquement spécifique, l'ethnopoétique élargit notre champ d'étude en y incorporant le langage comme acte social. Cette méthodologie nous permet d'étudier l'adaptation des sociétés traditionnelles aux nouvelles circonstances, par exemple, à l'arrivée des Blancs qui a bouleversé tout le continent du nouveau monde. A travers les deux optiques, le récit oral transcrit devient un vestige culturel qui nous permet de mieux percevoir les voix anciennes mais aussi la voix narrative du conteur et son contexte. La narratologie et la théorie de la performance ${ }^{16}$ rendent possible une distinction entre les structures historiques, formulaires et contextuels de façon à ce que les chercheurs de divers domaines puissent facilement échanger leurs données ethnographiques. Une nouvelle cohabitation entre l'anthropologie structurale française et l'ethnolinguistique américaine est donc aujourd'hui envisageable, voire souhaitable, alors que les voix se dissipent, non seulement par perte de références culturelles et linguistiques anciennes, mais aussi par manque d'attention sérieuse accordée aux voix non occidentales. 


\section{Claude Lévi-Strauss et Dell Hymes ont montré non seulement une fidélité méthodologique aux textes, mais aussi un profond respect pour ces voix et cultures interpellées. *}

* Manuscrit reçu en novembre 2003, accepté pour publication en mars 2004.

\section{NOTES}

1. Le travail le plus connu et le plus accessible aux non-spécialistes de la littérature est probablement Figures III de Gérard Genette dont la description des catégories temporelles, de la voix narrative et des perspectives seront appliquées dans notre analyse. Cependant, d'autres travaux ont été utilisés dans l'approche textuelle développée ici : notamment ceux de Uspenski (1972), de Bauman (1986), de Foley (1995), de Lecercle (1999) et de Bearth (1986) proposent des optiques et outils méthodologiques complémentaires à une démarche ethnopoétique.

2. Nous nous référons au travail de Foley (1995, p. 8 ; traduction de l'auteur C.M.) qui décrit le caractère contextuel de la performance et le nomme " arène de la performance » : « [...] le lieu dans lequel une forme spécialisée de la communication est autorisée ». Foley précise l'importance de cette conceptualisation pour l'étude poétique : «Dans une telle arène, les unités linguistiques - dans le cas du récit oral traditionnel, les divers "mots" ou unités d'énoncés qui constituent l'idiome - ne renvoient plus simplement aux significations littérales du langage quotidien extrinsèque à la performance, mais sont plutôt chargées de valeurs associées, particulières à l'événement qui a lieu ».

3. Pour la transcription du texte clackamas, nous avons suivi l'exemple de la transcription linguistique des textes de Victoria Howard édités par Melville Jacobs qui emploie les indicateurs américanistes contemporains pour les phonèmes en y ajoutant des signes d'expressivité littéraire. Les deux points ( :) indique, par exemple, le prolongement d'un son de voyelle, trait commun dans les récits clackamas. Pour les caractères qui ne sont pas disponibles sur certains PCs, nous avons suivi l'exemple de la transcription des textes clackamas adaptée par Dell Hymes (l'emploi du X majuscule, par exemple, pour marquer le son guttural indiqué normalement par un point placé sous le caractère) et dont les conventions sont expliquées dans la " Note orthographique » de In vain I tried to tell you.

4. La racine verbale -mqt est traduite par Howard comme "mourir». La non-finalité de cet événement indique que la mort peut avoir plusieurs sens pour les Chinooks. Or les personnages mythiques ne connaissent pas la mort telle qu'elle est vécue par les humains. Nous avons ici une problématique de nature métaphysique dans la pensée chinook qui mériterait attention.

5. Les particules marquées en lettres grasses ci-dessus ont des valeurs de conjonction adverbiale qui ont, pour certaines ( $k$ wálá et aga $a$ ), une notion temporelle.

6. Ces trois lignes sont soulignées dans la transcription avec une note qui dit "Kitsimanisays it thus, differently, whispered by Kitsimani " et un peu plus loin, " all whispered by her ». Dans le texte édité, Jacobs ajoute " whispering with obvious stupidity" ». Ces notes indiquent l'intonation que V. Howard a dû utiliser pour la voix de femme Grizzly.

7. V. Howard a précisé pendant la traduction que Femme Grizzly est restée à cet endroit pendant une seule journée.

8. La répétition est, bien évidemment, une forme de parallélisme qui a comme spécificité la différence au sein de la duplication au lieu de la régularisation que permettent la rime, le rythme et d'autres formes de parallélisme. Caractéristique de la poésie orale, la répétition nous propose un signe fondamental dans la création du sens poétique.

9. La métadiégèse consiste en toute instance de narration au sein d'un récit. Gérard Genette décrit le récit premier comme celui qui incorpore tout le discours narratif et le récit second comme celui intégré en tant qu'acte narratif à l'intérieur du texte global. Différents types de métadiégèse sont expliqués dans Figures III où Genette (1972, p. 245) nous précise que « tous ces jeux manifestent par l'intensité de leurs effets l'importance de la limite qu'ils s'ingénient à franchir au mépris de la 
vraisemblance, et qui est précisément la narration (ou la représentation) elle-même; frontière mouvante mais sacrée entre deux mondes : celui où l'on raconte, celui que l'on raconte ".

10. Un préfixe appositif étant cependant nécessaire pour l'accord du sujet avec le prédicat dans la syntaxe chinook, notre acteur devient "Askíntwa » au sein du récit.

11. Victoria Howard a aussi proposé comme titre Akitsimani Askintwa išdaquq, employant le sufixixe $A$ - à la place de $W a$ - pour les deux mères. Nous soulignons le fait que, dans les deux titres proposés, les femmes sont sur un pied d'égalité.

12. Dans la musique occidentale, la " note sensible " est située sur le septième degré de la gamme, un demi-ton au-dessous de la tonique qui est la note principale de la tonalité : « elle donne à l'oreille insatisfaite un sentiment de suspension qui appelle instamment la tonique, vers laquelle la sensible tend à monter d'un demi-ton » (Candé 1961, p. 224).

13. La traduction du verbe-IXam en langue française reste à résoudre. Le caractère intransitif de " dire » rend ce choix malheureux. "Interpeller » ou "saisir (son attention) verbalement " me semblent plus satisfaisants en ce qui concerne la mise en relation des interlocuteurs, connotée dans le verbe clackamas. La distinction de la transitivité du verbe anglais « tell » par rapport à « say » intransitif facilite la traduction des marqueurs de discours chinook en cette langue.

14. L'exigence verbale qui fait céder l'opposant est un motif récurrent dans la mythologie chinook. Nous trouvons de nombreux exemples aussi dans les textes de Victoria Howard.

15. Nous remarquons que, hormis ses propres métadiégèses et celui de Grue où elle figure comme public, le sujet principal de tous les récits insérés est Kitsimani.

16. Pour un bilan compréhensif des travaux théoriques sur le phénomène de la performance, voir Bauman et Briggs (1990).

\section{RÉFÉRENCES}

\section{BAUMAN Richard}

1986 Story, performance and event, Cambridge University Press, Cambridge.

BAUMAN Richard and Charles L. Briggs

1990 "Poetics and performance on language and social life », Annual Review of Anthropology, pp. 59-88.

\section{BEARTH Thomas}

1986 L'Articulation du temps et de l'aspect dans le discours toura, Peter Lang, Berne.

\section{BURKE Kenneth}

1931

1966 Language as symbolic action, University of California Press, Berkeley.

1973 The philosophy of literary form, University of California Press, Berkeley.

CANDÉ Roland de

1961 Dictionnaire de musique, Éditions du Seuil, Paris.

\section{CASSIRER Ernst}

1973 La Philosophie des formes symboliques. La pensée mythique, II, Éditions de Minuit, Paris [Philosophie der symbolischen Formen, Yale University Press, 1953].

\section{DÉsveAux Emmanuel}

1988 Sous le signe de l'ours. Mythes et temporalité chez les Ojibwa septentrionaux, Éditions de la Maison des sciences de l'homme, Paris. 
2001 Quadratura americana. Essai d'anthropologie lévi-straussienne, Georg, Genève.

FoLey John Miles

1995 The Singer of tales in performance, Indiana University Press, Bloomington.

GENETTE Gérard

1972 Figures III, Éditions du Seuil, Paris.

HyMES Dell

1981 "In vain I tried to tell you»: essays in Native American ethnopoetics, University of Pennsylvania Press, Philadelphia.

1985 "Language, memory, and selective performance, Cultee's "Salmon's myth" as twice told to Boas ", Journal of American Folklore, 98 (390), pp. 391-434, [réédité in Hymes, 2003, Now I know only so far : essays in ethnopoetics, University of Nebraska Press, Lincoln].

1987 "Anthologies and narrators », in Brian Swann and Arnold Krupat, eds, Recovering the word: essays on native american literature, Berkeley, University of California Press, pp. 41-84 [réédité in Hymes, 2003, Now I know only so far : essays in ethnopoetics, University of Nebraska Press, Lincoln].

HOPKINS G. M.

1959 Journals and papers, W. H. Gardner and N. H. Mackenzie, London.

JACOBS Melville

1958 Clackamas Chinook texts, Part I [CCT1], International Journal of American Linguistics, 24 (1), pt. 2., Research Center in Anthropology, Folklore and Linguistics, Publications 8, Indiana University, Bloomington.

1959a Clackamas Chinook texts, Part II [CCT2], International Journal of American Linguistics, 25 (2), pt. 2, Research Center in Anthropology, Folklore and Linguistics, Publication 11, Indiana University, Bloomington.

1959b The content and style of an oral literature: Clackamas Chinook myths and tales, The University of Chicago Press, Chicago

1960 The people are coming soon. Analyses of Clackamas Chinook myths and tales, University of Washington Press, Seattle.

JAKOBSON Roman

1973 Questions de poétique, Éditions du Seuil, Paris.

LECERCLE Jean-Jacques

1999 Interpretation as pragmatics, St Martin's Press Inc, New York.

Lévi-Strauss Claude

1964 Mythologiques. I. Le cru et le cuit, Plon, Paris.

1967 Mythologiques. II. Du miel aux cendres, Plon, Paris.

1968 Mythologiques. III. L'origine des manières de table, Plon, Paris.

1971 Mythologiques. IV. L'homme nu, Plon, Paris.

1987 «De la fidélité au texte », L'Homme, 101, XXVII (1), pp. 117-140.

Marx Karl

1982 Euvres. Philosophie, Gallimard, Paris, [1946]. 
MASON Catharine

1999 Méthodologies de l'ethnopoétique appliquées à l'art verbal de Victoria Howard, thèse présentée à l'université de Michel de Montaigne-Bordeaux III, Bordeaux.

RichaRd Jean-Pierre

1955 Poésie et profondeur, Éditions du Seuil, Paris.

TADIÉ Jean-Yves

1994 Le Récit poétique, Éditions Gallimard, Paris.

USPENSKY Boris

1972 A poetics of composition, University of California Press, Berkeley.

Voegelin C. F. et Thomas A. SEBEOK

1953 «Results of the conference of anthropologists and linguists », International Journal of American Linguistics, Memoir 8, 19 (2). 